\title{
Reflexiones y sentimientos desde un equipo de voluntarios del Ministerio de Salud y el Colegio Médico de Chile, después del terremoto reciente
}

\author{
MIGUEL O’RYAN G.
}

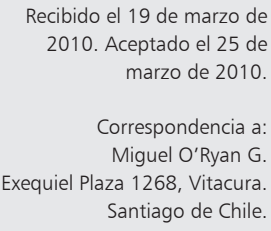

Recibido el 19 de marzo de Aceptado el 25 de Correspondencia a: Ryel Santiago de Chile.

\author{
Reflections and feelings from a team of \\ volunteers from the Ministry of Health \\ and the Chilean Medical Association, \\ after the recent earthquake
}

The experience of a group of 9 doctors and 6 nurses, most of them younger than 30 years of age, whom were part of the several volunteer groups directed towards the most damaged earthquake regions, is described. The team had to overcome a number of intense personal emotions related with the magnitude of the destruction, especially in the "adobe"-constructed houses and villages, in order to provide useful medical support. Moving out of the hospital setting, reaching out to the community in schools and emergency posts proved to be important in rural communities. An appropriate coordination of the volunteer groups, with the simultaneous action of municipal and state health authorities, together with well guided leadership, was critical for an effective response in the larger city of Talcahuano/Hualpén. Within the second week of the aftermath, acute respiratory and intestinal infections were the most common medical complaints together with intense -in many cases severe- emotional distress associated mostly with fear to after shakes ("replicas"), tsunami, and social unrest. The severe earthquake that struck Chile has left many lessons for the future that will need to be analyzed seriously and with the conviction that effective and timely prevention of catastrophic aftermath consequences, although costly, must be a key element of the country's development plan. More importantly, the hundreds if not thousands of volunteers from a variety of health related professions that were moved by the scenes of suffering, and whom responded to individual or group initiatives, allow to foresee that the nation has the moral stamina required to overcome the tragedy and become a better society.

(Rev Med Chile 2010; 138: 270-273).

Keywords: Chile; Disasters; Earthquakes; Students, health occupations; Volunteers.
E l sábado 13 de marzo regresó a Santiago el Grupo 1 de voluntarios, reunidos ante el llamado del Colegio Médico de Chile: nueve médicos y seis enfermeras, quienes compartimos 8 días de intensas emociones en Licantén (Maule) y Hualpén (Talcahuano). Habíamos partido a esa zona el viernes 5 de marzo, luego de una reunión de organización básica el día anterior, con la misión de ser autosustentables, pacientes ante las posibles fallas de organización, dispuestos a ayudar sin condiciones, y flexibles para modificar nuestras tareas de acuerdo con evaluaciones diarias en los 
sitios de trabajo. Como "la antigüedad constituye grado", los 15 jóvenes que conformaban el grupo me designaron espontáneamente su coordinador, o "comandante", como cariñosamente me denominaron parodiando un programa de televisión, por ser bastante mayor que ellos. Al partir, el viernes en la mañana, cada uno iba cargado con sus propias emociones y muchas incertidumbres: " $i$ a dónde vamos, doc?” "¿dónde alojaremos, profe?”, “¿habrá agua?", respuestas que por cierto yo no tenía, ya que la instrucción era contactar al Director de Salud en el Regimiento de Talca para recibir nuestra misión. La fuerza, el entusiasmo, el ánimo, la alegría se percibieron desde el primer momento, en el bus. Varios se conocían previamente, la mayoría recién egresados de Universidades públicas o privadas. Salvo dos, eran menores de 30 años. Junto con el grupo iban dos voluntarios argentinos encargados de armar un hospital de campaña en Curicó; se habían desconectado de su unidad por un problema de descoordinación y estaban a la deriva, buscando su unidad. Espero que Leandro y Gustavo hayan finalmente podido cumplir a cabalidad la misión que tan generosamente los trajo a nuestro país.

Las primeras emociones profundas de sobrecogimiento surgieron al entrar a Talca. Si tuviera que resumirlo, parecía una ciudad bombardeada, con severo compromiso en prácticamente todas las cuadras que íbamos pasando. En ese momento cesaron las "tallas" y los chistes que distendían el viaje para dar inicio a profundas sensaciones internas y el deseo intenso de querer ayudar lo más rápidamente posible. La base de operaciones -el Regimiento de Talca- se encontraba bajo una actividad febril, con un gimnasio transformado en un conjunto de unidades operativas, incluyendo Salud. Entre quienes ya estaban en el lugar se percibía mucho compromiso personal y de esfuerzo: subsecretarios, diputados, autoridades municipales y regionales entraban y salían en un ambiente obviamente militarizado. Luego de algunas dudas, se asignó nuestra "unidad" al pueblo de Licantén para apoyar a los equipos de Salud más adentro del borde costero; la información -que en mi impresión era algo dispersa en ese momento- sugería que ésta era la mejor destinación, ya que el borde costero se encontraba cubierto por otros grupos voluntarios, fruto de iniciativas municipales o personales. La segunda emoción fuerte surgió al pasar por Curepto, un hermoso pueblo construido prácticamente en su totalidad con adobe: la ma- yoría de sus casas estaban destruidas. Llegamos a Licantén cerca de las 23 horas, a un hospital sólido, con electricidad, aunque sin agua, pero un lujo comparado con nuestras aprehensiones. La información de su Director, quien se preocupó con gentileza de nuestra instalación, era que el hospital estaba plenamente funcionante, con uno de sus cuatro médicos damnificados, el equipo de salud cansado y emocionalmente tenso. Las consultas espontáneas en la urgencia no habían aumentado, incluso en algunos días habían disminuido. Los principales problemas de salud en esta etapa, a una semana del terremoto, se estimaban asociados al hacinamiento, la falta de agua, el temor y angustia de las personas. La conclusión fue que había que salir a terreno para evaluar el estado de salud de la población, especialmente los pacientes de mayor edad con enfermedades crónicas, personas con hogares destruidos y ofrecer tratamiento y apoyo emocional. Se diseñó un plan en conjunto con el Director y el equipo local, para implementar atención primaria en tres localidades rurales. Se requería transporte, lo que representaba una de las principales dificultades por superar. Parte del equipo apoyaría el trabajo en el hospital y otra parte iría a apoyar a la localidad vecina de Hualañé, cuyo hospital fue severamente dañado. A pesar del largo viaje, el equipo preparó esa misma noche un operativo que resultó en la implementación al día siguiente de uno de los tres centros de atención primaria y la preparación de otros dos (se utilizaron dos colegios y una posta de urgencia) para el día subsiguiente. El apoyo de la enfermera local, de mujeres líderes comunitarias y la radio local fueron claves para habilitar los centros y llamar a la población a acudir a ellos. Se identificaron y visitaron, además, casas con pacientes postrados. Se atendieron en total cerca de 120 personas, de todas las edades, aunque en su mayoría ancianos. La población se encontraba angustiada y temerosa, pero con disposición para salir adelante. Las infecciones respiratorias y las diarreas agudas, junto con las enfermedades crónicas en necesidad de tratamiento, eran los principales problemas médicos que requerían atención. Cada uno de los equipos, conformados por parejas de médicos recientemente egresados y enfermeras, cumplió una labor que puedo catalogar con una sola palabra: "notable". Demostraron sin excepción un muy buen nivel de conocimiento, un marcado profesionalismo en la atención y, lo que más me impresionó, una 
humanidad en el trato que permitió que fueran importantes agentes de contención y apoyo emocional para los pacientes. El pago que no se nos olvidará nunca, especialmente a estos jóvenes profesionales, era un "que Dios los bendiga". Las noches eran para preparar la comida, repasar y comentar las actividades del día que terminaba y programar las del día siguiente. Se requería de iniciativa, otra virtud que demostró tener cada uno de los integrantes del equipo, especialmente las enfermeras, para definir cuáles eran los campos de acción en que fuéramos más útiles para la comunidad. En un momento hubo que sobrepasar una cuota de desánimo, por la sensación de algunos de no estar aprovechando toda nuestra capacidad de acción. Las reuniones del grupo, con expresión libre aunque dirigidas y ordenadas, recogiendo opiniones francas, maduras, con profundas reflexiones personales, algunas cargadas con un importante componente emocional, fueron clave para sortear los momentos difíciles. La evaluación del trabajo del fin de semana fue que las necesidades médicas asociadas al terremoto estaban relativamente cubiertas y que el grupo local podría cubrir las contingencias futuras. Se tomó la decisión de trasladar al grupo a un área de mayor necesidad; la instrucción recibida del Ministerio de Salud fue responder al llamado de Hualpén, que requería con urgencia apoyo en diferentes localidades. Un grupo semejante al nuestro se encontraba apoyando a Talcahuano.

El grupo se instaló en el moderno, aún no inaugurado Centro de Salud Familiar (CESFAM) de Hualpencillo. La habilitación del Centro para la atención de urgencia había ya sido realizada por otro grupo de voluntarios, en los días previos y habían sido redestinados a Talcahuano; vaya un agradecimiento a ellos por su excelente trabajo. En estrecha colaboración con las autoridades de Salud Regional y de Salud Municipal de Hualpén se diseñó un plan que incluía atención completa en tres centros de Salud, incluyendo el CESFAM Hualpencillo, un Centro de Salud Comunitario y un centro vecinal, y apoyo al equipo médico de dos CESFAM para descomprimir y permitir la salida a terreno de equipos locales. En el transcurso de los cinco días el grupo se consolidó, logrando una rica interacción con los abnegados equipos de salud local. La maravilla de Hualpén fue comprobar la tremenda solidaridad de tantos voluntarios con deseos de aportar. Nuestra casa transitoria se fue llenando rápidamente con equipos multiprofesionales de psicólogos, salud mental, antropólogos, geógrafos, veterinarios, todos comprometidos en la empresa común de aportar ayuda. Al momento de nuestra despedida, éramos más de 30 los que habitábamos en el CESFAM compartiendo techo, cocina, baños, experiencias, vivencias y emociones. Se logró una organización efectiva que permitía esta cohabitación en forma armoniosa y amistosa. Para lograr una acción efectiva en el área de salud, resultó trascendental el trabajo ejecutivo y coordinado de la autoridad de Salud. Todos los días había reunión del Comité de Emergencia liderado por el Director de Salud del área, con presencia de las autoridades de Salud de las dos comunas, la autoridad sanitaria de la Armada, una persona responsable de la coordinación de los diversos grupos voluntarios en la zona y ambos coordinadores de los voluntarios en Talcahuano y Hualpén. Pude constatar en terreno la solidaridad del Perú, al visitar su Hospital de Campaña implementado en el hipódromo, unidad de alta complejidad que fue redestinada, gracias a la flexibilidad de todos, a la atención primaria que concentraba las mayores necesidades. Un apoyo significativo provino de un grupo voluntario del Servicio de Atención Médica de Urgencia (SAMU) de Antofagasta, que hizo expedito el traslado de pacientes graves. El apoyo del Ejército también fue muy importante, destinando soldados para nuestra protección (lo que fue conveniente durante los primeros dos días, cuando había algo de inquietud en algunos integrantes del grupo) y ofreciendo el traslado de regreso en avión para todo el equipo, lo que hizo muy expedita esa operación. Un nuevo momento de sobrecogimiento fue la visita del grupo al centro de Talcahuano. Constatar cómo la fuerza del mar había movido barcos de gran tonelaje como naipes de una baraja, nos impresionó profundamente. La frecuente repetición de temblores ("réplicas") y la alerta de "tsunami" el día jueves 11 de marzo produjeron una marcada tensión y angustia en los habitantes de la ciudad y el equipo debió sobreponerse a sus propios temores para seguir trabajando con eficiencia. La racionalidad no estaba siempre presente, como se ejemplifica en personas subiendo a los cerros vecinos al CESFAM a pesar de estar a más de 7 kilómetros de la costa. La necesidad de implementar un plan de apoyo emocional para los funcionarios de Salud y la población se hizo patente y fue estimulante constatar que la auto- 
ridad Ministerial está trabajando en un Plan de Salud Mental para las áreas afectadas, con metas al corto, mediano y largo plazo.

Al final de nuestra estadía, cumpliéndose dos semanas del terremoto, pudimos concluir que la ayuda de voluntarios es deseable por al menos un mes, para apoyar a los equipos locales mientras la ciudadanía se recupera. Los equipos médicos voluntarios atenderán patologías propias de la atención primaria, especialmente infecciones respiratorias que irán en aumento, diarreas agudas asociadas a la falta de agua potable, hacinamiento, eliminación inadecuada de excretas, heridas infectadas y, por sobre todo, alteraciones transitorias y en algunos casos crónicas del ánimo (pánico, depresión). La puesta en marcha de un programa especial de vacunas, el abastecimiento de medicamentos en cantidades necesarias para el aumento de las patologías descritas y la implementación del Programa de Salud Mental, son acciones que pondrán a prueba a las nuevas autoridades de salud.

La experiencia vivida por nuestro grupo de voluntarios ha sido única para nosotros y siento que todos los que participamos en ella la recordaremos de por vida. El terremoto nos mostró de manera violenta que tenemos mucho por mejorar y ello debe ser motivo de un análisis serio y sos- tenido en el tiempo. La prevención es muy cara, pero debemos ser capaces de diseñar programas eficientes, aunque nos duela el bolsillo, que deben mantenerse actualizados y evaluados en forma protocolizada para que puedan responder eficaz y oportunamente, aunque fueran pocas las veces en que se los requiera. Pero, quizás más importante, nos demostró que nuestra población puede contar con personas profundamente comprometidas con su país y sus compatriotas. En el área de la Salud y otras, jóvenes y no tan jóvenes, médicos, enfermeras, kinesiólogos, tecnólogos, dentistas, psicólogos, psiquiatras, geógrafos, veterinarios, bomberos, las Fuerzas Armadas y de Orden, y muchos otros profesionales y técnicos, de todo el país, se dieron de verdad la mano para ayudar en la medida de sus posibilidades a nuestros hermanos en necesidad. Quisiera brindar mi reconocimiento a tantos voluntarios que, mantenidos en el anonimato (como debe ser el voluntariado), dejaron todo lo suyo pendiente para responder al llamado de la solidaridad. Gracias a ambas Carolinas, a Juan Manuel, Enzo, Alexandra, Víctor, Julio, Carlos, Marcela, María Paz, Nicole, Katy, Fabián y Paulina. No cabe ninguna duda que el país, gracias a muchas personas como ustedes, tiene la fortaleza moral para salir adelante. 